\title{
Multicenter Validation of Mean Upper Cervical Cord Area Measurements from Head 3D T1-Weighted MR Imaging in Patients with Multiple Sclerosis
}

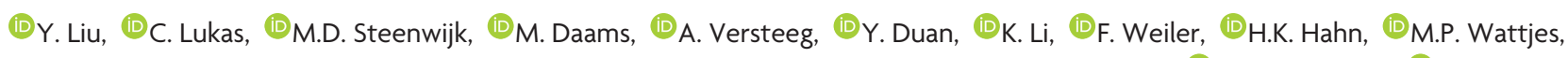
(1)F. Barkhof, and ${ }^{\circ} \mathrm{H}$. Vrenken 을

\begin{abstract}
BACKGROUND AND PURPOSE: Spinal cord atrophy is a common and clinically relevant characteristic in multiple sclerosis. We aimed to perform a multicenter validation study of mean upper cervical cord area measurements in patients with multiple sclerosis and healthy controls from head MR images and to explore the effect of gadolinium administration on mean upper cervical cord area measurements.
\end{abstract}

MATERIALS AND METHODS: We recruited 97 subjects from 3 centers, including 60 patients with multiple sclerosis of different disease types and 37 healthy controls. Both cervical cord and head 3D T1-weighted images were acquired. In 11 additional patients from 1 center, head images before and after gadolinium administration and cervical cord images after gadolinium administration were acquired. The mean upper cervical cord area was compared between cervical cord and head images by using intraclass correlation coefficients (ICC) for both consistency (ICC consist) and absolute (ICC $\left.\mathrm{Cbs}_{\text {s }}\right)$ agreement.

RESULTS: There was excellent agreement of mean upper cervical cord area measurements from head and cervical cord images in the entire group $\left(I C C_{a b s}=0.987\right)$ and across centers and disease subtypes. The mean absolute difference between the mean upper cervical cord area measured from head and cervical cord images was $2 \mathrm{~mm}^{2}$ (2.3\%). Additionally, excellent agreement was found between the mean upper cervical cord area measured from head images with and without gadolinium administration $\left(\right.$ ICC $\left._{\mathrm{abs}}=0.991\right)$ and between the cervical cord and head images with gadolinium administration $\left(\right.$ ICC $\left._{\mathrm{abs}}=0.992\right)$.

CONCLUSIONS: Excellent agreement between mean upper cervical cord area measurements on head and cervical cord images was observed in this multicenter study, implying that upper cervical cord atrophy can be reliably measured from head images. Postgadolinium head or cervical cord images may also be suitable for measuring mean upper cervical cord area.

ABBREVIATIONS: EDSS = Expanded Disability Status Scale; $\mathrm{Gd}=$ gadolinium; $\mathrm{HC}=$ healthy control; $\mathrm{ICC}=$ intraclass correlation coefficient; $\mathrm{ICC}_{\mathrm{abs}}=\mathrm{ICC}$ absolute agreement; $I_{C C}$ consist $=I C C$ consistency; $M U C C A=$ mean upper cervical cord area; PPMS = primary-progressive MS; RRMS = relapsing-remitting MS, SPMS = secondary-progressive MS

S pinal cord atrophy is recognized as a common and clinically relevant characteristic in patients with multiple sclerosis. ${ }^{1}$ Differences in cervical cord volume and area among patients

Received July 18, 2015; accepted after revision August 27.

From the Department of Radiology (Y.L., Y.D., K.L.), Xuanwu Hospital, Capital Medical University, Beijing, P.R. China; Department of Radiology and Nuclear Medicine (Y.L., M.D.S., M.D., A.V., M.P.W., F.B., H.V.), Neuroscience Campus Amsterdam; Department of Anatomy and Neurosciences (M.D.), Section of Clinical Neuroscience; and Department of Physics and Medical Technology (H.V.), Neuroscience Campus Amsterdam, VU University Medical Center, Amsterdam, the Netherlands; Department of Neurology and Tianjin Neurological Institute (Y.L.), Tianjin Medical University, General Hospital, Tianjin, P.R. China; Department of Diagnostic and Interventional Radiology and Nuclear Medicine (C.L.), St. Josef Hospital, Ruhr University, Bochum, Germany; and Fraunhofer MEVIS, Institute for Medical Image Computing (F.W., H.K.H.), Bremen, Germany.

This work was supported by the European Committee for Treatment and Research in Multiple Sclerosis/Magnetic Research Imaging in Multiple Sclerosis Fellowship from the European Committee for Treatment and Research in Multiple Sclerosis (Y.L), the National Science Foundation of China (No. 81101038 and 81501736), Beijing with different phenotypes of MS and healthy controls (HCs) measured by MR imaging have been identified in many studies. $^{2-4}$ Furthermore, a modest or strong correlation between spinal cord atrophy and disability has been demonstrated in

Municipal Administration of Hospitals Clinical Medicine Development of Special Funding Support (No. ZYLX201609), the Beijing Natural Science fund (No. 7133244), the Beijing Nova Programme (No. xx2013045), and the Key Projects in the National Science \& Technology Pillar Program during the Twelfth Five-Year Plan Period (No. 2012BAI10B04). Part of this work was supported by the German Federal Ministry for Education and Research, Bundesministerium für Bildung und Forschung, and the German Competence Network Multiple Sclerosis (Kompetenznetz Multiple Sklerose) (No. 01GI0914). The VUmc MS Center Amsterdam is sponsored through the program grant by the Dutch MS Research Foundation (Nos. 09-358d and 14-358e). Please address correspondence to Yaou Liu, MD, PhD, Department of Radiology, Xuanwu Hospital, Capital Medical University, Beijing 100053, China; e-mail: asiaeurope80@gmail.com

- Indicates open access to non-subscribers at www.ajnr.org

三 Indicates article with supplemental on-line table.

http://dx.doi.org/10.3174/ajnr.A4635 
numerous studies, suggesting that spinal cord atrophy is an essential determinant of clinical disability and a potential outcome measure to monitor MS disease progression. ${ }^{5-7}$ The measurement of the upper cervical cord area is a well-established method for the assessment of spinal cord atrophy and has been applied in most studies so far. ${ }^{2,3,5,8,9}$ Both image acquisition and spinal cord segmentation are technically feasible and more accurate in the upper cervical region compared with other parts of the cord or the entire cord. ${ }^{10}$ In addition, the upper cervical cord is more frequently affected by MS pathology than lower parts of the spinal cord. ${ }^{11}$

Mean upper cervical cord area (MUCCA) can be measured by using 3D T1-weighted MR images of the cervical cord. ${ }^{3,4}$ The MUCCA also has been measured recently on 3D T1weighted MR images of the head covering the upper cervical cord, which has yielded promising results showing associations between MUCCA and clinical disability and disease progression. $^{2,5}$ Measuring the MUCCA from head MR images offers the opportunity to analyze MUCCA retrospectively in datasets without dedicated cervical 3D T1-weighted images, and it can reduce costs and patient burden in prospective studies by eliminating the need for separate cervical cord image acquisitions if these are only acquired to measure the MUCCA. An MR imaging contrast agent is commonly used to detect the bloodbrain barrier breakdown and inflammation in new lesions ${ }^{12,13}$ in patients with MS, which might influence the MUCCA measurements by tissue-contrast changes. The effect of the MR imaging contrast agent on MUCCA measurement also has to be investigated to ease the implementation of MUCCA as an auxiliary measurement in clinical practice. Although MUCCA measurements based on head 3D T1-weighted images have been successfully used in a monocenter study, ${ }^{14}$ multicenter validation is lacking. In addition, the possible effect of intravenous contrast administration on MUCCA measurements has not been investigated.

Therefore, the aim of the current study was to validate the measurement of the MUCCA on the basis of head compared with cervical cord 3D T1-weighted images in patients with MS and healthy controls on different MR imaging systems by using different acquisition parameters from multiple centers and to explore the effect of gadolinium (Gd) administration on MUCCA measurements.

\section{MATERIALS AND METHODS \\ Subjects}

The study was approved by the local institutional review board of the 3 centers (Amsterdam, Beijing and Bochum), and informed consent was obtained from each participant.

To investigate the variability of the MUCCA based on head 3D T1 MR images and cervical cord 3D T1 MR images, we selected scans from ongoing local cohorts in which separate head and cervical cord 3D T1 MR images were acquired. All images were checked for visibility of the spinal cord without artifacts, and head images were checked for coverage of the upper cervical cord. A total of 97 subjects were recruited from 3 centers (A: Amsterdam, B: Beijing, and C: Bochum), including 40 patients with relapsing-remitting MS (RRMS), 10 with
Table 1: Scan protocols for all 3 centers $^{a}$

\begin{tabular}{|c|c|c|c|}
\hline & $\begin{array}{c}\text { Center A } \\
(n=58)\end{array}$ & $\begin{array}{c}\text { Center B } \\
(n=28)\end{array}$ & $\begin{array}{c}\text { Center C } \\
(n=7)\end{array}$ \\
\hline Vendor & GE Healthcare ${ }^{b}$ & Siemens $^{c}$ & Philips Healthcare ${ }^{d}$ \\
\hline Scanner & Signa HDxt & Trio Tim & Achieva \\
\hline \multicolumn{4}{|l|}{ Head MRI } \\
\hline Sequence & FSPGR & MPRAGE & FFE \\
\hline No. of sections & 176 & 176 & 180 \\
\hline $\mathrm{TR}(\mathrm{ms})$ & 7.8 & 1600 & 10 \\
\hline TE (ms) & 3 & 2.13 & 4.6 \\
\hline $\mathrm{TI}$ (ms) & 450 & 1000 & 1000 \\
\hline $\mathrm{FA}$ & $12^{\circ}$ & $9^{\circ}$ & $8^{\circ}$ \\
\hline Voxel size (mm) & $0.94 \times 0.94 \times 1$ & $1 \times 1 \times 1$ & $1 \times 1 \times 1$ \\
\hline \multicolumn{4}{|l|}{ Cervical MRI } \\
\hline Sequence & FSPGR & MPRAGE & FFE \\
\hline No. of sections & 176 & 96 & 64 \\
\hline $\mathrm{TR}(\mathrm{ms})$ & 7.3 & 2000 & 8 \\
\hline TE (ms) & 3 & 3.36 & 3.5 \\
\hline TI (ms) & 450 & 1100 & 1000 \\
\hline $\mathrm{FA}$ & $15^{\circ}$ & $10^{\circ}$ & $8^{\circ}$ \\
\hline Voxel size (mm) & $1 \times 1 \times 1$ & $1 \times 1 \times 1$ & $1 \times 1 \times 1$ \\
\hline
\end{tabular}

Note:-FA indicates flip angle; FFE, fast-field echo; FSPGR, fast spoiled gradient recalled.

${ }^{a}$ All images had a sagittal orientation.

${ }^{\mathrm{b}}$ Milwaukee, Wisconsin.

cErlangen, Germany.

${ }^{\mathrm{d}}$ Best, the Netherlands.

primary-progressive MS (PPMS), 10 with secondary-progressive MS (SPMS), and 37 HCs. The subjects from the Amsterdam center were selected from a larger cohort (most data from this cohort have been published before ${ }^{3}$ ) in a semirandom fashion, to include 20 with RRMS, 10 with SPMS, 10 with PPMS, and 20 HCs. We ensured that the selected subsets spanned the range of spinal cord area of the total group (on the basis of the previously published measurements from cervical images $^{3}$ ).

MS diagnosis was determined according to the 2010 revisions of the McDonald criteria. ${ }^{15}$ The main demographic and clinical characteristics included the Expanded Disability Status Scale (EDSS) ${ }^{16}$ scores and disease duration of the participants.

To explore the effect of intravenous Gd administration on MUCCA measurements, we included an additional 11 patients from Center C, including 2 with clinically isolated syndrome suggestive of MS and 9 with RRMS. In these patients, head images before and after Gd administration and cervical cord images after Gd administration were acquired.

\section{MR Imaging Acquisition}

In all 3 centers, a 3T MR imaging system was used to acquire high-resolution 3D sagittal T1-weighted head (with head coils) and cervical cord (with spine coils) images in the same scanning session. Corrections for geometric distortion due to gradient nonlinearity were applied in 3D. Details of MR imaging systems and acquisition parameters are provided in Table 1 .

\section{Image Analysis}

Images were anonymized before MUCCA measurement and presented in a random order to the rater (Y.L., with $>8$ years of experience). MUCCA was defined as the average area of the 30-mm-length section of the upper cervical cord, starting at the upper borders of vertebral level C2, measured by using the 
semiautomated software NeuroQLab (Fraunhofer MEVIS, Bremen, Germany) (Fig 1). In short, NeuroQLab segments the upper cervical cord from surrounding non-spinal cord tissue by using a Gaussian mixture modeling method. The workflow and the reliability of the software have been described previously. ${ }^{2,3,17,18}$

\section{Statistical Analysis}

Analyses were performed by using SPSS software (Version 18; IBM, Armonk, New York). Kolmogorov-Smirnov tests were performed together with visual inspection of histograms to assess the normality of the variables. Comparison of the demographic data and MR imaging parameters between different disease subtypes and HCs or among different centers was conducted by using ANOVA, with Bonferroni correction for post hoc comparisons.
The intraclass correlation coefficients (ICCs) were calculated to assess both absolute agreement $\left(\mathrm{ICC}_{\mathrm{abs}}\right.$ ) and consistency $\left(\mathrm{ICC}_{\text {consist }}\right.$ ) between MUCCA measurements from head and cervical cord images. These calculations were also performed separately for each center and each disease subtype. The difference between MUCCA measurements on head and cervical cord images was calculated in square millimeters and as percentages. For the exploratory analysis of the effects of intravenous Gd administration, the above-mentioned analyses were additionally performed in patients with MS from Center $\mathrm{C}$ included in that part of our study.

\section{RESULTS \\ Demographics and Clinical and MR Imaging Characteristics}

Five subjects were excluded from the final analysis, including 2 HCs from Center A ( 1 head scan and 1

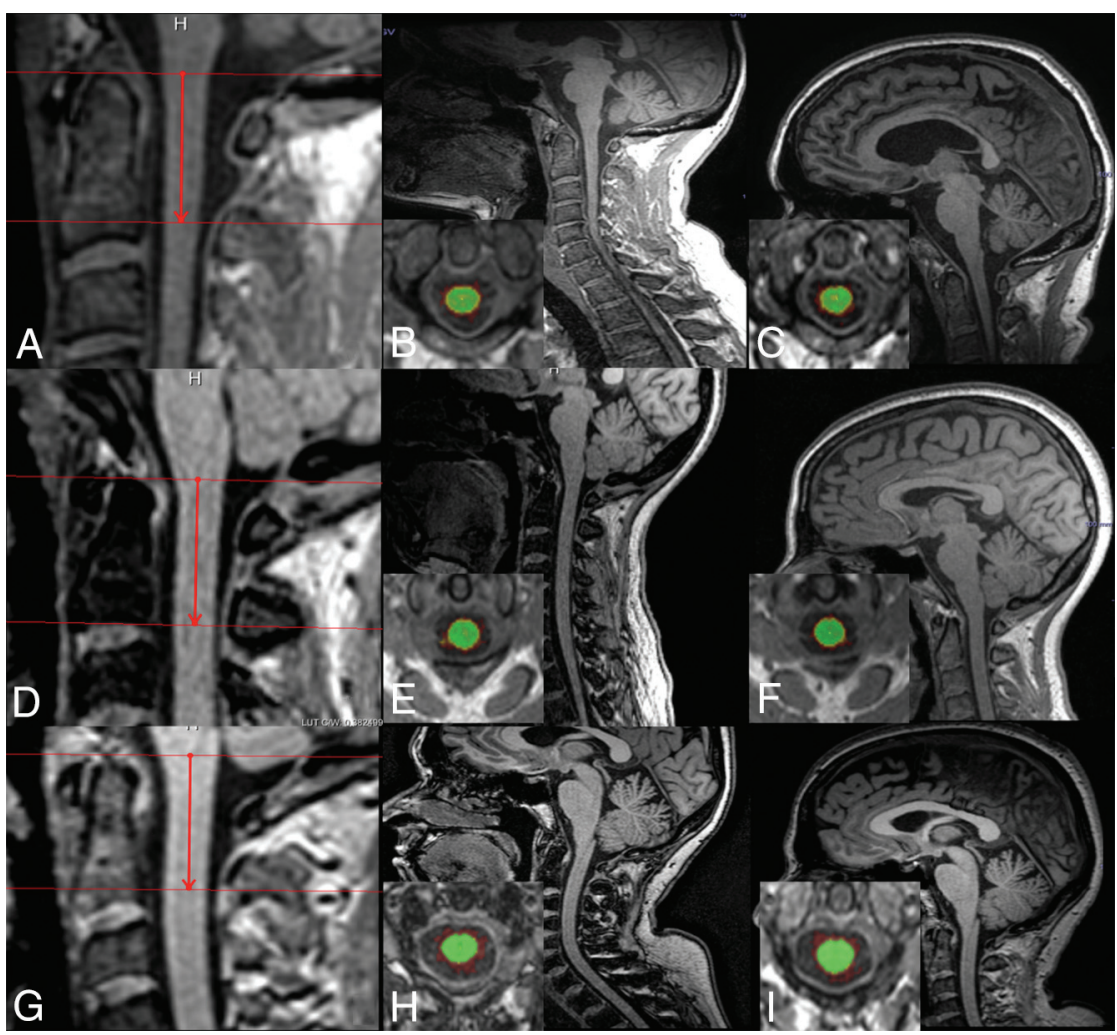
cervical cord scan with artifacts) and 1 with RRMS, 1 HC from Center B (head scan with artifacts), and 1 patient with RRMS from Center C (the head scan without Gd administration did not cover the upper cervical cord). Finally, 103 subjects were left for final analysis, including 93 subjects without Gd administration from 3 centers (Table 2) and 10 patients with images before and after Gd administration from Center C. As shown in Table 2 and in line with the recruitment of long-standing patients with MS and matched healthy controls in Center $\mathrm{A},{ }^{3}$ the age of the subjects (including patients and HCs) was older in Center A than in the other 2 centers, and patients from center A had a longer average disease duration and higher EDSS scores than patients from Center B.

MUCCA measured from cervical cord images did not differ among centers in HCs (Center A: $81 \pm 9 \mathrm{~mm}^{2}$, Center B: $80 \pm 6 \mathrm{~mm}^{2}$, Center C: $84 \pm$ $\left.10 \mathrm{~mm}^{2}, F=0.51, P=.61\right)$. For the whole cohort, the MUCCA measured from cervical cord images in patients with MS was significantly lower than that in HCs $(P<.001)$. Specifically, in patients with SPMS $(P=.02)$ and PPMS $(P<.001)$, the MUCCA was
FIG 1. Examples of images from each center (cervical cord and head images). Sagittal T1-weighted MR image of the ROI selection (30-mm section length) in the upper cervical cord, starting at the upper borders of vertebral level C2 $(A, D$, and $G)$, and sagittal images of the cervical cord and head with representative axial reformats overlaid by corresponding segmentation images from a patient with SPMS from Center A: B, MUCCA cervical cord images $=62 \mathrm{~mm}^{2}, C$, MUCCA head images $=64 \mathrm{~mm}^{2}$. A patient with RRMS from Center B: $E$, MUCCA cervical cord images $=74 \mathrm{~mm}^{2}$; $F$, MUCCA head images $=75 \mathrm{~mm}^{2}$. An HC subject from Center C: $H$, MUCCA cervical cord images $=82 \mathrm{~mm}^{2} ;$ I, MUCCA head images $=80 \mathrm{~mm}^{2}$.

Table 2: Demographic and clinical characteristics of subjects without Gd administration ${ }^{\text {a }}$

\begin{tabular}{|c|c|c|c|c|c|c|c|}
\hline & \multicolumn{4}{|c|}{ Center A $(n=58)$} & \multicolumn{2}{|c|}{ Center B $(n=28)$} & \multirow{2}{*}{$\begin{array}{c}\text { Center C }(n=7) \\
\text { HC }(n=7)\end{array}$} \\
\hline & $\operatorname{RRMS}(n=20)$ & SPMS $(n=10)$ & PPMS $(n=10)$ & HC $(n=18)$ & RRMS $(n=19)$ & HC $(n=9)$ & \\
\hline Sex (female/male) & $12: 8$ & $4: 6$ & $5: 5$ & $11: 7$ & $13: 6$ & $6: 3$ & $5: 2$ \\
\hline Age (yr) & $50.5 \pm 8.4(33-65)$ & $63.4 \pm 4.7(54-70)$ & $55.8 \pm 4.6(49-65)$ & $51.9 \pm 6.8(36-61)$ & $33.5 \pm 8.7$ (17-49) & $32.4 \pm 11.9(21-56)$ & $38.6 \pm 11.8(26-53)$ \\
\hline Disease duration (yr) & $18.8 \pm 5.8(9-29)$ & $20.7 \pm 7.6(10-33)$ & $19.5 \pm 4.6(12-27)$ & NA & $4.3 \pm 2.5(1-10)$ & NA & NA \\
\hline EDSS score & $3.5(2.5-6.5)$ & $5(3.5-8)$ & $5.5(3-7.5)$ & NA & $3(0-6.5)$ & NA & NA \\
\hline
\end{tabular}

Note:-NA indicates not applicable.

${ }^{a}$ Data are presented as mean \pm SD (range). 


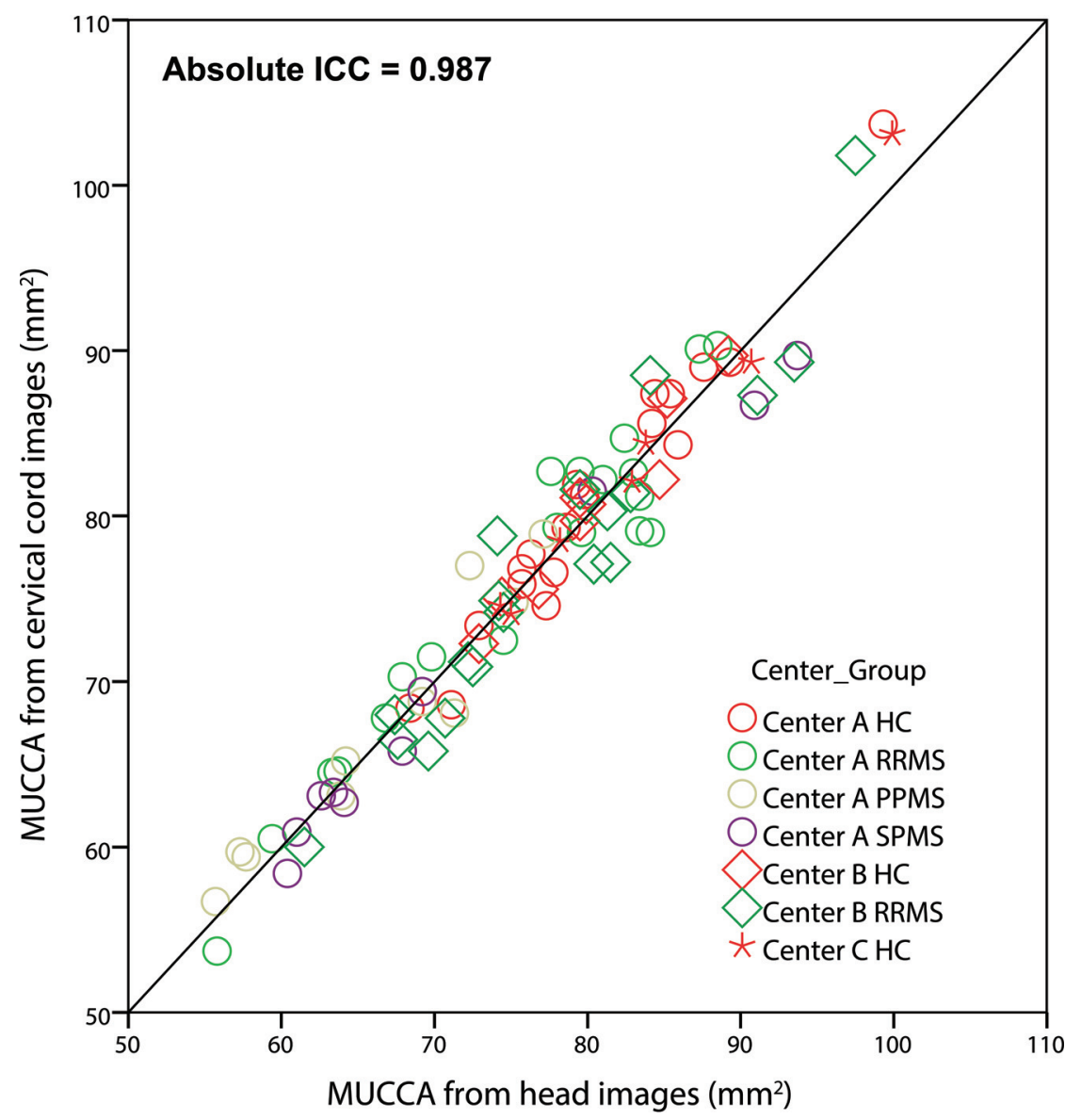

FIG 2. Scatterplot of the MUCCA measured from head-versus-cervical cord images. Different shapes indicate different centers (circle: Center A; diamond: Center B; star: Center C), and different colors represent different subgroups of subjects (red: HC; green: RRMS; yellow: PPMS; purple: SPMS). The solid black line indicates perfect agreement.

significantly smaller compared with HCs, and patients with PPMS showed smaller MUCCAs than those with RRMS ( $P=$ $.01)$, whereas the MUCCA did not differ between patients with RRMS and HCs $(P=.20)$ or between those with SPMS and those with RRMS $(P=.63)$. MUCCA measured from head images showed similar group effects across centers and groups, including differences between those with MS and HCs, as well as among disease subtypes.

\section{Concordance of MUCCA Measurements from Cervical Cord Images and Head Images}

There was excellent absolute and consistency agreement of MUCCA measurements from head and cervical cord images in the whole group $\left(\mathrm{ICC}_{\mathrm{abs}}=0.987, \mathrm{ICC}_{\mathrm{consist}}=0.987\right)$. Similarly, strong ICCs were observed across different centers and disease subtypes (On-line Table). The mean difference between MUCCA measured from head images and cervical cord images was 0.1 $\mathrm{mm}^{2}(0.1 \%)$, ranging from $-5 \mathrm{~mm}^{2}(-6.1 \%)$ to $5 \mathrm{~mm}^{2}(6.6 \%)$. The mean absolute difference was $2 \mathrm{~mm}^{2}(2.3 \%)$, ranging from 0 to $5 \mathrm{~mm}^{2}(6.6 \%)$ (On-line Table). For illustration purposes, scatterplots (Fig 2) and Bland-Altman plots (Fig 3) were created, which provide information on the interchangeability of the 2 measurements, by using MUCCAs from cervical cord images as the criterion standard.

\section{The Effect of Gd Administration on MUCCA Measurements}

Table 3 presents the results of the comparisons between MUCCA measurements based on 3D T1 MR images with and without Gd administration. Excellent agreement was found between MUCCA measurements on head images with and without Gd administration $\left(\mathrm{ICC}_{\mathrm{abs}}=0.991, \mathrm{ICC}_{\text {consist }}=0.990\right)$ and between MUCCA measurements on cervical cord images and head images with Gd enhancement $\left(\mathrm{ICC}_{\mathrm{abs}}=0.992\right.$, $\left.\mathrm{ICC}_{\text {consist }}=0.992\right)$.

\section{DISCUSSION}

The results of the current study demonstrate that MUCCA can be reliably measured by using head images covering the upper cervical cord in a multicenter setting across MS subtypes. Furthermore, our data suggest that Gd enhancement does not have a substantial influence on MUCCA measurements.

Upper cervical cord atrophy is regarded as an important biomarker correlating with clinical disability and predicting disease progression in MS. ${ }^{2,4,5}$ Measurement of MUCCA is commonly used for assessing upper spinal cord atrophy. In our study, MUCCA was measured by using semiautomated software, for which high intra- and interrater reproducibility has been observed for MUCCA measurements either from head images ${ }^{3}$ or cervical cord images $^{2}$ in previous studies. Although the primary objective of the current study was to determine agreement between head and cervical images, it is reassuring that in our study, differences were found in MUCCAs among disease subtypes: Patients with PPMS and SPMS showed smaller MUCCAs compared with HCs, and patients with PPMS demonstrated smaller MUCCAs compared with those with RRMS, based on both cervical cord and head images. For Center A, MUCCA has been reported before for these subjects because they were part of a larger cohort; in that study, MUCCA was measured independently by another rater than the one performing the measurements in the current study. ${ }^{3}$ These current findings are in line with previous studies showing that cervical cord atrophy is more pronounced in progressive subtypes. $^{2,4}$

Some previous studies have used head images to measure cervical cord atrophy, ${ }^{2,5,8,9}$ and a viable method is measuring the MUCCA. However, for MUCCA measurements to be widely applied to head images, in particular in a clinical trial setting, validation in a multicenter setting is necessary. Our study showed an average absolute difference in MUCCA between cervical cord and head images of $<4 \%$, and ICCs for absolute agreement $\geq 0.978$ across centers and disease subtypes, which is consistent with a previous study from a single center showing an ICC $>0.95$ be- 


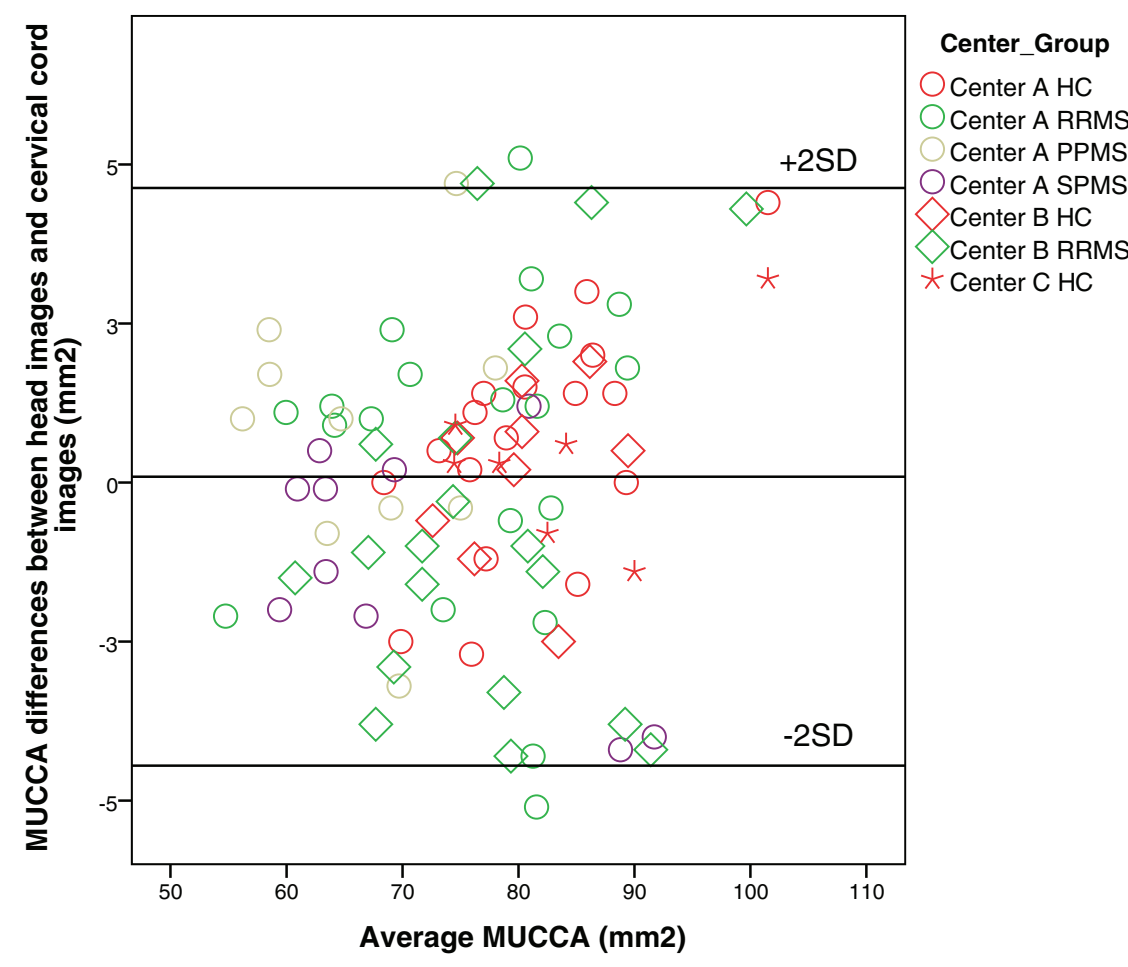

FIG 3. Bland-Altman plots for MUCCA measurements from cervical cord images and head images. The averages of MUCCA measurements on cervical cord and head images are shown on the horizontal axis, and the differences in MUCCA between the 2 measurements are shown on the vertical axis. Different shapes indicate different centers (circle: Center A; diamond: Center B; star: Center C), and different colors represent different subgroups of subjects (red: HC; green: RRMS; yellow: PPMS; purple: SPMS).

Table 3: Demographic and clinical characteristics of patients with Gd administration from Center $C$ and comparison between MUCCA from nonenhanced head images, enhanced cervical cord and head images ${ }^{a}$

\begin{tabular}{|c|c|}
\hline & Center C (8 RRMS + 2 CIS) \\
\hline Sex (female/male) & $6: 4$ \\
\hline Age $(y r)$ & $39.0 \pm 10.8(29-67)$ \\
\hline Disease duration (yr) & $1.2 \pm 1.2(0.1-2.9)$ \\
\hline EDSS score & $2.6 \pm 1.7(1-5.5)$ \\
\hline Gd + MUCCA (cervical cord images) $\left(\mathrm{mm}^{2}\right)$ & $82 \pm 10(67-96)$ \\
\hline Gd-MUCCA (head images) $\left(\mathrm{mm}^{2}\right)$ & $82 \pm 10(66-99)$ \\
\hline $\mathrm{Gd}+$ MUCCA (head images) $\left(\mathrm{mm}^{2}\right)$ & $0.82 \pm 0.10(66-99)$ \\
\hline \multicolumn{2}{|l|}{$\begin{array}{l}\mathrm{Gd}+\text { MUCCA (head images) vs } \\
\text { Gd+ MUCCA (cervical cord images) }\end{array}$} \\
\hline Difference $\left(\mathrm{mm}^{2}\right)$ & $-0.17 \pm 2.00(-30-30)$ \\
\hline$\%$ Difference & $-0.28 \pm 2.21(-3.48-3.12)$ \\
\hline Absolute difference $\left(\mathrm{mm}^{2}\right)$ & $1.49 \pm 1.25(0-30)$ \\
\hline$\%$ Absolute difference & $1.71 \pm 1.32(0.48-3.48)$ \\
\hline $\mathrm{ICC}_{\text {consist }}$ & 0.990 \\
\hline$I C C_{a b s}$ & 0.991 \\
\hline \multicolumn{2}{|l|}{$\begin{array}{l}\text { Gd }+ \text { MUCCA (head images) vs } \\
\text { Gd-MUCCA (head images) }\end{array}$} \\
\hline Difference $\left(\mathrm{mm}^{2}\right)$ & $-034 \pm 1.89(-2-4)$ \\
\hline$\%$ Difference & $-0.40 \pm 2.05(-2.61-4.42)$ \\
\hline Absolute difference $\left(\mathrm{mm}^{2}\right)$ & $1.36 \pm 1.29(0-4)$ \\
\hline$\%$ Absolute difference & $1.53 \pm 1.34(0.14-4.42)$ \\
\hline $\mathrm{ICC}_{\text {consist }}$ & 0.992 \\
\hline$I C C_{a b s}$ & 0.992 \\
\hline
\end{tabular}

Note:-CIS indicates clinically isolated syndrome.

${ }^{a}$ Data are presented as mean \pm SD (range).

tween MUCCA measurements from head and cervical cord images in patients with RRMS. ${ }^{14}$ The excellent agreement between MUCCA measurements using cervical cord and head images without systematic shift indicates that measuring the upper cervical cord area is feasible by using head MR imaging datasets if they cover the upper cervical cord and if gradient inhomogeneity correction is applied in 3D. Retrospective studies can thus be performed in large MS data bases investigating upper cervical cord atrophy by using head images, which may help to improve our understanding of the role of spinal cord atrophy in MS. For future studies, upper cervical cord atrophy is an accessible outcome measure in treatment trials and clinical research without additional cervical cord image acquisitions, thus providing additional information without increasing image acquisition cost or patient burden. ${ }^{19}$

Gd administration is often included in routine MR imaging protocols of patients with $\mathrm{MS}^{20}$ and can change the contrast of brain and spinal cord images. The question of whether the MUCCA can be reliably measured on images obtained after Gd administration is therefore clinically relevant, but it has not been investigated before, to our knowledge. In the current study, we were unable to perform a rigorous assessment with available data for only 10 patients from a single center. However, these preliminary results showed excellent agreement of the MUCCA between head images with or without Gd administration and between head and cervical cord images with Gd administration, suggesting that Gd administration has little effect on MUCCA measurement. These results should be confirmed in a larger group of patients and a multiscanner setting. If future studies confirm the current finding, one could use head images or cervical cord images after Gd administration to measure MUCCA, which would present another option to assess cervical cord atrophy. However, care should be taken in case of enhancing intramedullary cervical cord lesions that may affect MUCCA estimation on images obtained after Gd administration. Because in the present study no enhancing lesions were observed on the postcontrast images, we cannot exclude a bias in such cases. Furthermore, enhanced spinal nerves after Gd administration may influence the segmentation of the cord, another potential factor influencing MUCCA measurement.

Although this study included only 3 centers, the 3 main MR imaging vendors and the most commonly used sequences for high-resolution cervical cord and head imaging were included. Despite the (subtle) differences in tissue contrast among different scanners and 3D T1 sequences, agreement was excellent, suggesting the generalizability of our results beyond scanners and pulse sequences. Therefore the MUCCA has the potential to become a viable marker for MS in a routine clinical setting and clinical treatment trials.

Several limitations apply to this work. First, we did not normalize the MUCCA measurements for intracranial volume, body height, or other parameters because optimal parameters for cer- 
vical cord volume or area normalization remain controversial. $^{21-23}$ Second, the presence of cord lesions including T2 hyperintensity and $\mathrm{T} 1$ hypointensity in the measurement area may influence MUCCA measurements due to underestimation of white matter volumes owing to lesion misclassification. ${ }^{9}$ However, the lesions are present on both cervical cord and head images, which may cause a similar effect on MUCCA measurements. The high ICC values in all subgroups suggest that if an effect is present, it is comparable between both image types. Third, HCs were younger than those with MS, especially those with SPMS and PPMS in Center A in the current study. High ICC values were identified in HCs and all MS subgroups, suggesting that age has little influence on the consistency of measurements of the MUCCA between cervical cord and head images. Finally, this is a cross-sectional study; a longitudinal study is warranted to extrapolate the current findings to MUCCA changes with time.

\section{CONCLUSIONS}

Excellent agreement between MUCCA measurements from cervical cord and head images was observed across centers and disease subtypes in a multicenter setting, implying that upper cervical cord atrophy can be reliably measured by using head images covering the upper cervical cord. If there are no enhancing cervical cord lesions, postcontrast 3D T1-weighted images of the cervical cord or head may also be a suitable source for measuring MUCCA. These findings have important implications for clinical studies, including treatment trials.

Disclosures: Carsten Lukas—UNRELATED: Biogen Idec, Bayer Schering, Novartis, Sanofi, Genzyme, and Teva; Employment: endowed professorship supported by the Novartis Foundation*; Grants/Grants Pending: Bayer Schering, Teva, and Merck Serono*; Payment for Lectures (including service on Speakers Bureaus): Biogen Idec, Bayer Schering, Novartis, Sanof, Genzyme, and Teva; Travel/Accommodations/ Meeting Expenses Unrelated to Activities Listed: Bayer Schering; OTHER: Dr Carsten Lukas received consulting and speaker's honoraria from Biogen Idec, Bayer Schering, Novartis, Sanofi, Genzyme, and Teva and a research scientific grant support from Bayer Schering, Teva, and Merck Serono. He holds an endowed professorship supported by the Novartis Foundation. Martin D. Steenwijk—RELATED: Grant: Dutch MS Research Foundation (grant No. 09-358d).* Marita Daams—RELATED: Grant: Dutch MS Research Foundation (grant No. 09-358d), ${ }^{*}$ Comments: private sponsorship to the VUmc Center Amsterdam which is sponsored through a program grant by the Dutch MS Research Foundation (grant No. 09-358d). Mike P. Wattjes-UNRELATED: Consultancy: Roche, ${ }^{*}$ Novartis*; Payment for Lectures (including service on Speakers Bureaus): Biogen; Royalties: Springer; OTHER: Dr Mike P. Wattjes serves as a consultant for Biogen Idec and Roche. Frederik Barkhof_-RELATED: Grant: Dutch MS Research Foundation*; UNRELATED: Consultancy: Synthon, Teva, Roche, Biogen Idec, Novartis; OTHER: Dr Frederik Barkhof serves as a consultant for Bayer Schering Pharma, Sanofi-Aventis, Biogen Idec, Teva, Merck Serono, Novartis, Roche, Synthon, and Jansen Research. Hugo Vrenken-UNRELATED: European Committee for Treatment and Research in Multiple Sclerosis/Magnetic Research Imaging in Multiple Sclerosis, Merck Serono, Novartis, Teva; Dutch MS Research Foundation, Comments: Dr Yaou Liu and I obtained a European Committee for Treatment and Research in Multiple Sclerosis application, and I was designated as the supervisor. The work described in the current article was performed during the time he was at our institution and employed through this fellowship; research grant for multiple sclerosis brain imaging; pending research grant for multiple sclerosis brain imaging; research grants for multiple sclerosis brain imaging; OTHER: Dr Hugo Vrenken has received research funding from Novartis, Pfizer, and Merck Serono and speaker honoraria from Novartis. * Money paid to the institution.

\section{REFERENCES}

1. Lycklama G, Thompson A, Filippi M, et al. Spinal-cord MRI in multiple sclerosis. Lancet Neurol 2003;2:555-62 CrossRef Medline

2. Lukas C, Sombekke MH, Bellenberg B, et al. Relevance of spinal cord abnormalities to clinical disability in multiple sclerosis: MR imag- ing findings in a large cohort of patients. Radiology 2013;269:542-52 CrossRef Medline

3. Daams M, Weiler F, Steenwijk MD, et al. Mean upper cervical cord area (MUCCA) measurement in long-standing multiple sclerosis: relation to brain findings and clinical disability. Mult Scler 2014;20: 1860-65 CrossRef Medline

4. Rocca MA, Horsfield MA, Sala S, et al. A multicenter assessment of cervical cord atrophy among MS clinical phenotypes. Neurology 2011;76:2096-102 CrossRef Medline

5. Lukas C, Knol DL, Sombekke MH, et al. Cervical spinal cord volume loss is related to clinical disability progression in multiple sclerosis. J Neurol Neurosurg Psychiatry 2015;86:410-18 CrossRef Medline

6. Bonati U, Fisniku LK, Altmann DR, et al. Cervical cord and brain grey matter atrophy independently associate with long-term MS disability. J Neurol Neurosurg Psychiatry 2011;82:471-72 CrossRef Medline

7. Losseff NA, Webb SL, O'Riordan JI, et al. Spinal cord atrophy and disability in multiple sclerosis: a new reproducible and sensitive MRI method with potential to monitor disease progression. Brain 1996;119(pt 3):701-08 CrossRef Medline

8. Engl C, Schmidt P, Arsic M, et al. Brain size and white matter content of cerebrospinal tracts determine the upper cervical cord area: evidence from structural brain MRI. Neuroradiology 2013;55: 963-70 CrossRef Medline

9. Biberacher V, Boucard CC, Schmidt P, et al. Atrophy and structural variability of the upper cervical cord in early multiple sclerosis. Mult Scler 2015;21:875-84 CrossRef Medline

10. Klein JP, Arora A, Neema M, et al. A 3T MR imaging investigation of the topography of whole spinal cord atrophy in multiple sclerosis. AJNR Am J Neuroradiol 2011;32:1138-42 CrossRef Medline

11. Bot JC, Barkhof F. Spinal-cord MRI in multiple sclerosis: conventional and nonconventional MR techniques. Neuroimaging Clin $N$ Am 2009;19:81-99 CrossRef Medline

12. Gaitan MI, Shea CD, Evangelou IE, et al. Evolution of the bloodbrain barrier in newly forming multiple sclerosis lesions. Ann $\mathrm{Neu}$ rol 2011;70:22-29 CrossRef Medline

13. Miller DH, Rudge $P$, Johnson G, et al. Serial gadolinium enhanced magnetic resonance imaging in multiple sclerosis. Brain 1988; 111(pt 4):927-39 CrossRef Medline

14. Liu Y, Wang J, Daams M, et al. Differential patterns of spinal cord and brain atrophy in NMO and MS. Neurology 2015;84:1465-72 CrossRef Medline

15. Polman CH, Reingold SC, Banwell B, et al. Diagnostic criteria for multiple sclerosis: 2010 revisions to the McDonald criteria. Ann Neurol 2011;69:292-302 CrossRef Medline

16. Kurtzke JF. Rating neurologic impairment in multiple sclerosis: an expanded disability status scale (EDSS). Neurology 1983;33:1444-52 CrossRef Medline

17. Lukas C, Hahn HK, Bellenberg B, et al. Sensitivity and reproducibility of a new fast 3D segmentation technique for clinical MR-based brain volumetry in multiple sclerosis. Neuroradiology 2004;46: 906-15 CrossRef Medline

18. Lukas C, Hahn HK, Bellenberg B, et al. Spinal cord atrophy in spinocerebellar ataxia type 3 and 6: impact on clinical disability. J Neurol 2008;255:1244-49 CrossRef Medline

19. Barkhof F, Simon JH, Fazekas F, et al. MRI monitoring of immunomodulation in relapse-onset multiple sclerosis trials. $\mathrm{Nat} \mathrm{Rev} \mathrm{Neu-}$ rol 2012;8:13-21 CrossRef Medline

20. Rovaris M, Filippi M. Contrast enhancement and the acute lesion in multiple sclerosis. Neuroimaging Clin N Am 2000;10:705-16, viii-ix Medline

21. Zivadinov R, Banas AC, Yella V, et al. Comparison of three different methods for measurement of cervical cord atrophy in multiple sclerosis. AJNR Am J Neuroradiol 2008;29:319-25 CrossRef Medline

22. Healy BC, Arora A, Hayden DL, et al. Approaches to normalization of spinal cord volume: application to multiple sclerosis. J Neuroimaging 2012;22:e12-19 CrossRef Medline

23. Oh J, Seigo M, Saidha S, et al. Spinal cord normalization in multiple sclerosis. J Neuroimaging 2014;24:577-84 CrossRef Medline 